\title{
Carter and Gaschütz theories beyond soluble groups
}

\section{Milagros Arroyo-Jordá ${ }^{1}$ Paz Arroyo-Jordá ${ }^{1}$. Rex Dark ${ }^{2}$ - Arnold D. Feldman ${ }^{3}$. María Dolores Pérez-Ramos ${ }^{4}$}

Received: 19 May 2021 / Accepted: 19 January 2022 / Published online: 11 February 2022

(c) The Author(s) 2022

\begin{abstract}
Classical results from the theory of finite soluble groups state that Carter subgroups, i.e. self-normalizing nilpotent subgroups, coincide with nilpotent projectors and with nilpotent covering subgroups, and they form a non-empty conjugacy class of subgroups, in soluble groups. This paper presents an extension of these facts to $\pi$-separable groups, for sets of primes $\pi$, by proving the existence of a conjugacy class of subgroups in $\pi$-separable groups, which specialize to Carter subgroups within the universe of soluble groups. The approach runs parallel to the extension of Hall theory from soluble to $\pi$-separable groups by Čunihin, regarding existence and properties of Hall subgroups.
\end{abstract}

Keywords Finite soluble groups $\cdot \pi$-separable groups $\cdot$ Carter subgroups $\cdot$ Hall systems Mathematics Subject Classification 20D10 · 20D20

\section{María Dolores Pérez-Ramos}

Dolores.Perez@uv.es

Milagros Arroyo-Jordá

marroyo@mat.upv.es

Paz Arroyo-Jordá

parroyo@mat.upv.es

Rex Dark

rex.dark@nuigalway.ie

Arnold D. Feldman

afeldman@fandm.edu

1 Escuela Técnica Superior de Ingeniería Industrial, Instituto Universitario de Matemática Pura y Aplicada IUMPA, Universitat Politècnica de València, Camino de Vera, s/n, 46022 Valencia, Spain

2 School of Mathematics, Statistics and Applied Mathematics, National University of Ireland, University Road, Galway, Ireland

3 Franklin and Marshall College, Lancaster, PA 17604-3003, USA

4 Departament de Matemàtiques, Universitat de València, C/ Doctor Moliner 50, 46100 Burjassot, València, Spain 


\section{Introduction}

All groups considered are finite.

The well-known result of Carter [7] states that each soluble group possesses exactly one conjugacy class of self-normalizing nilpotent subgroups (the so-called Carter subgroups). His discovery was of interest at the time not only by analogy with Cartan subalgebras, but also by sharing the flavour of Hall's result on the existence and conjugacy of Hall $\rho$ subgroups, for all sets of primes $\rho$, in every finite soluble group. Indeed, classical results of Hall theory state that soluble groups are characterized by the existence of Hall $\rho$-subgroups for all sets of primes $\rho$ (Hall [15, 16]). Cleverly and beautifully, Gaschütz [12] unifies both families of subgroups under the concept of $\mathfrak{F}$-covering subgroups associated to saturated formations $\mathfrak{F}$, by considering the class $\mathfrak{S}_{\rho}$ of soluble $\rho$-groups, and the class $\mathfrak{N}$ of nilpotent groups, respectively, as very particular cases of saturated formations. This is the origin of the theory of distinguished conjugacy classes of subgroups in finite soluble groups related to certain classes of groups, which quickly splits into the theories of covering subgroups and projectors related to Schunck classes and formations, and the dual theory of injectors and Fitting classes. (See Definitions 3.1, 3.2.) We refer to the excellent monographs [5, 9] for an account of developments on the topic in the universes of soluble and finite groups. On the other hand, if $\pi$ is a set of primes, $\pi$-separable groups have Hall $\pi$-subgroups, and also every $\pi$-subgroup is contained in a conjugate of any Hall $\pi$-subgroup, by a well-known result of Čunihin [6]. However, there has been no successful extension of Carter and Gaschütz' results to $\pi$-separable groups corresponding to the extension of Hall's results to $\pi$-separable groups by Čnihin. The aim of this paper is to provide such an extension. Note that for non-soluble groups, the known equivalence of Carter subgroups, $\mathfrak{N}$-covering subgroups and $\mathfrak{N}$-projectors no longer holds. In arbitrary finite groups $\mathfrak{N}$-projectors do always exist (cf. [9, III. Theorem (3.10)]), though they do not form a conjugacy class of subgroups in general, and the existence of $\mathfrak{N}$-covering subgroups is not guaranteed. For Carter subgroups no counterpart has been found for a general non-soluble group, though they are conjugate when existing, as finally settled by Vdovin in [19]. Within the theory of $\mathfrak{F}$-normalizers, initiated by Carter and Hawkes, there appear extensions of classical embedding properties of subgroups, such as $\mathfrak{F}$-subnormal subgroups, associated to saturated formations $\mathfrak{F}$ (cf. [8, 17], [9, Chapter V], [5, Chapters 4, 6]). In [2] a definition of $\mathfrak{F}$-normality in soluble groups consistent with the lattice properties of $\mathfrak{F}$-subnormal subgroups is achieved. This concept is applied in this paper to extend Carter subgroups taking heed of its very definition, as nilpotent self-normalizing subgroups, and enables us to address the lack of a Carter and Gaschütz counterpart in the extension of Hall theory from soluble groups to $\pi$-separable groups. The deep knowledge of techniques and progress now achieved in this area allow us to reach the results of this paper. We notice that a group $G$ is soluble if and only if $G$ is $\rho$-separable for all sets of primes $\rho$. It is an easy but motivating observation that a group is $\pi$-separable if and only if it is $\rho$-separable for suitable sets of primes related to the set of primes $\pi$, as stated in Proposition 2.1. It is then straightforward to provide an extension of the existence of Hall systems of soluble groups to $\pi$-separable groups, as shown in Sect. 2 . At the end of that section we discuss to what extent the existence of these extended Hall systems characterizes $\pi$-separability. This setting suggests the consideration of classes $\mathfrak{N}^{\pi}$ of groups which are a direct product of a $\pi$-group by a nilpotent $\pi^{\prime}$-group, where $\pi^{\prime}$ stands for the complement of $\pi$ in the set $\mathbb{P}$ of all prime numbers, to play the role of the class of nilpotent groups. In Sect. 3, we present briefly the general framework from the theory of soluble groups and classes of groups which relates to our purposes, focusing on some concepts and preliminary 
results which are crucial to establish our main results; particularly, we introduce and adjust the above-mentioned extension of normality for saturated formations according to our needs. The main results of the paper are then carried out in Sect. 4. For a set of primes $\pi$, we prove that if $G$ is a $\pi^{\prime}$-soluble group, then the $\mathfrak{N}^{\pi}$-projectors coincide with the $\mathfrak{N}^{\pi}$-covering subgroups and they form a non-empty conjugacy class of subgroups (Theorem 4.5). (Notice that, by the Feit-Thompson theorem, for any set of primes $\rho$, a $\rho$-separable group is either a $\rho$-soluble group or a $\rho^{\prime}$-soluble group, so that the hypothesis of $\pi^{\prime}$-solubility means no real restriction but more a question of adjusting the sets of primes; see Remark 4.1). Besides, Theorem 4.14 proves that in a $\pi^{\prime}$-soluble group, $\mathfrak{N}^{\pi}$-projectors, and so also $\mathfrak{N}^{\pi}$-covering subgroups, can be described and characterized as a family of subgroups which specialize to Carter subgroups within the universe of soluble groups. Finally, it is remarkable that Carter subgroups are the cornerstone for the existence and conjugacy of injectors associated to Fitting classes in soluble groups. In a forthcoming paper [4], our Carter-like subgroups are used to generalize these results to $\pi$-separable groups.

\section{From soluble to $\pi$-separable groups}

As mentioned in the Introduction, we pursue an extension of the theory of finite soluble groups to the universe of $\pi$-separable groups, $\pi$ a set of primes. With this aim we analyze first the reach of $\pi$-separability further from the universe of soluble groups. We refer to [14] for basic results on $\pi$-separable groups, and to [9] for background on classes of groups; we shall adhere to their notations. If $\pi$ is a set of primes, let us recall that a group $G$ is $\pi$-separable if every composition factor of $G$ is either a $\pi$-group or a $\pi^{\prime}$-group. We start by noticing that a group $G$ is soluble if and only if it is $\rho$-separable for all sets of primes $\rho$. Regarding $\pi$-separability, $\pi$ a set of primes, it is clearly equivalent to $\pi^{\prime}$-separability, so that there is no loss of generality to assume that $2 \in \pi$. Then, by the Feit-Thompson theorem, a $\pi$-separable group is $\pi^{\prime}$-soluble, i.e. the group is $\pi^{\prime}$-separable with every $\pi^{\prime}$-composition factor a $p$-group for some prime $p \in \pi^{\prime}$. The following extension for $\pi$-separable groups is easily proved:

Proposition 2.1 For a group $G$, if $2 \in \pi \subseteq \mathbb{P}$ the following statements are pairwise equivalent:

1. $G$ is $\pi$-separable;

2. $G$ is $\rho$-separable for every set of primes $\rho$ such that either $\pi \subseteq \rho$ or $\pi \cap \rho=\emptyset$;

3. $G$ is $\pi^{\prime}$-separable $\left(\pi^{\prime}\right.$-soluble $)$.

Remark 2.2 The need of the hypothesis $2 \in \pi$ for the validity of the equivalences in Proposition 2.1 is clear. Certainly, for a group $G$ and any set of primes $\pi$, it holds that $2 \rightarrow 1 \leftrightarrow 3$. But statement 2 implies that the $\pi^{\prime}$-compositions factors of the group $G$ are soluble. Then, by the Feit-Thompson theorem, if $2 \notin \pi$, statement 2 is equivalent to the solubility of the group $G$ and $1 \nrightarrow 2$ in general.

Consequently, by Proposition 2.1, if $2 \in \pi \subseteq \mathbb{P}$, every $\pi$-separable group possesses a Sylow $p$-complement of $G$, i.e. a Hall $p^{\prime}$-subgroup of $G$, for each $p \in \pi^{\prime}$, as well as a Hall $\pi^{\prime}$-subgroup, and these are pairwise permutable subgroups with coprime indices in the group. In analogy with [9, I. Definitions (4.1), (4.5), (4.7)] it appears to be natural now to introduce the following concepts, which are proven to hold in $\pi$-separable groups if $2 \in \pi$, by the previous comment and as explained below: 
Definition 2.3 Let $G$ be a group and $\pi$ be a set of primes.

$\mathbf{K}_{\pi}$ : A complement $\pi$-basis of $G$ is a set $\mathbf{K}_{\pi}$ containing exactly one Sylow $p$-complement of $G$, i.e. a Hall $p^{\prime}$-subgroup of $G$, for each $p \in \pi^{\prime}$, and exactly one Hall $\pi^{\prime}$-subgroup.

$\Sigma_{\pi}$ : A Hall $\pi$-system of $G$ is a set $\Sigma_{\pi}$ of Hall subgroups of $G$ satisfying the following two properties:

1. For each set of primes $\rho$ such that either $\pi \subseteq \rho$ or $\pi \cap \rho=\emptyset, \Sigma_{\pi}$ contains exactly one Hall $\rho$-subgroup.

2. If $H, K \in \mathbf{\Sigma}_{\pi}$, then $H K=K H$.

$\mathbf{B}_{\pi}$ : A Sylow $\pi$-basis of $G$ is a set $\mathbf{B}_{\pi}$ of subgroups of $G$ satisfying the following two properties:

1. $\mathbf{B}_{\pi}$ contains exactly one Hall $\pi$-subgroup, and exactly one Sylow $p$-subgroup for each $p \in \pi^{\prime}$.

2. If $H, K \in \mathbf{B}_{\pi}$, then $H K=K H$.

Obviously these systems may not exist in arbitrary groups. By the previous comments, if the group is $\pi$-separable and $2 \in \pi$, then complement $\pi$-bases do exist.

Note also that Sylow $\pi$-systems and complement and Sylow $\pi$-bases are hereditary with respect to normal subgroups and factor groups.

For any set $\rho$ of primes and a group $G$, we denote by $\operatorname{Hall}_{\rho}(G)$ the set of all Hall $\rho$ subgroups of $G$. If $p$ is a prime, then $\operatorname{Syl}_{p}(G)$ will denote the set of all Sylow $p$-subgroups of $G$. We keep mimicking the exposition in [9, I. Sect. 4]. The arguments there are easily adapted to prove the following corresponding results.

Proposition 2.4 [9, I. Proposition (4.4)] Assume that the group $G$ has a complement $\pi$-basis, say $\mathbf{K}_{\pi}$ (particularly, if the group $G$ is $\pi$-separable and $2 \in \pi \subseteq \mathbb{P}$ ). If $\rho$ is a set of primes such that $\pi \subseteq \rho$, let $G_{\rho}=\bigcap\left\{X \mid X \in \operatorname{Hall}_{p^{\prime}}(G) \cap \mathbf{K}_{\pi}, p \in \rho^{\prime} \subseteq \pi^{\prime}\right\}$. On the other hand, if $\rho$ is a set of primes such that $\pi \cap \rho=\emptyset$, let $G_{\rho}=\bigcap\left\{X \mid\left(X \in \operatorname{Hall}_{p^{\prime}}(G) \cap \mathbf{K}_{\pi}, p \in\right.\right.$ $\left.\left.\rho^{\prime} \cap \pi^{\prime}\right) \vee\left(X \in \operatorname{Hall}_{\pi^{\prime}}(G) \cap \mathbf{K}_{\pi}\right)\right\}$. Then

1. $\Sigma_{\pi}:=\left\{G_{\rho} \mid(\pi \subseteq \rho \subseteq \mathbb{P}) \vee(\rho \subseteq \mathbb{P}, \rho \cap \pi=\emptyset)\right\}$ is a Hall $\pi$-system of $G$, and

2. $\boldsymbol{\Sigma}_{\pi}$ is the unique Hall $\pi$-system of $G$ containing $\mathbf{K}_{\pi}$.

We shall say that $\mathbf{\Sigma}_{\pi}$ is the Hall $\pi$-system generated by the complement $\pi$-basis $\mathbf{K}_{\pi}$.

Corollary $2.5[9$, I. Corollary (4.6)] Let $G$ be a $\pi$-separable group, $2 \in \pi \subseteq \mathbb{P}$. Then there is a bijective map between the set of all complement $\pi$-bases and the set of all Hall $\pi$-systems of $G$, such that to each complement $\pi$-basis corresponds the Hall $\pi$-system generated by it, and conversely, to each Hall $\pi$-system corresponds the complement $\pi$-basis contained in it.

On the other hand, it is clear that every Hall $\pi$-system contains a unique Sylow $\pi$-basis. Also, each Sylow $\pi$-basis generates a unique Hall $\pi$-system, by taking the product of the suitable elements in the basis to construct each element in the Hall $\pi$-system. We can easily state also the following result:

Corollary 2.6 [9, I. Lemma (4.8)] Let $G$ be a $\pi$-separable group, $2 \in \pi \subseteq \mathbb{P}$. Then there is a bijective map between the set of all Hall $\pi$-systems and the set of all Sylow $\pi$-bases of $G$, such that to each Hall $\pi$-system corresponds the Sylow $\pi$-basis contained in it, and conversely, to each Sylow $\pi$-basis corresponds the Hall $\pi$-system generated by it as described above. 
If $\mathbf{K}_{\pi}$ is a complement $\pi$-basis of a group $G$ and $g \in G$, it is clear that $\mathbf{K}_{\pi}^{g}:=\left\{X^{g} \mid X \in\right.$ $\left.\mathbf{K}_{\pi}\right\}$ is again a complement $\pi$-basis of $G$, and this defines an action by conjugation of $G$ on the set of all complement $\pi$-bases of $G$. Analogously, any group $G$ acts by conjugation on the set of all its Hall $\pi$-systems, and also on the set of all its Sylow $\pi$-bases.

By Proposition 2.1 again we have that if $G$ is a $\pi$-separable group, $2 \in \pi \subseteq \mathbb{P}$, then $G$ acts transitively on the set of all Hall $p^{\prime}$-subgroups for every $p \in \pi^{\prime}$, as well as on the set of all Hall $\pi^{\prime}$-subgroups, and the following result also holds.

Theorem 2.7 [9, I. Theorems (4.9), (4.10), (4.11), Corollary (4.12)] Let G be a $\pi$-separable group, $2 \in \pi$. Then:

1. The number of Hall $\pi$-systems of $G$ is $\prod_{S \in \mathbf{K}_{\pi}}\left|G: N_{G}(S)\right|$, where $\mathbf{K}_{\pi}$ is a complement $\pi$-basis of $G$.

2. The group $G$ acts transitively by conjugation on the set of all complement $\pi$-bases, on the set of all Hall $\pi$-systems, as well as on the set of all Sylow $\pi$-bases.

One might wish that the existence of Hall $\pi$-systems in finite groups would characterize $\pi$-separability, but this is not the case, even assuming the transitive action of the group by conjugation on the set of all Hall $\pi$-systems. The alternating group of degree 5 together with the set $\pi=\{2,3\}$ is a counterexample. A characterization of $\pi$-separability by the existence of Hall subgroups had been in fact given by Du [10], as shown in the next result. For notation, for a group $G$ and any set of primes $\rho$, the group $G$ is said to satisfy $E_{\rho}$ if $G$ has a Hall $\rho$-subgroup; if in addition each $\rho$-subgroup is contained in the conjugate of a Hall $\rho$-subgroup, it is said that $G$ satisfies $D_{\rho}$.

Theorem 2.8 [10, Theorems 1, 3] For a group $G$ and a set of primes $\pi$, the following statements are pairwise equivalent:

(i) $G$ is $\pi$-separable.

(ii) G satisfies:

1. $E_{\pi}$ and $E_{\pi^{\prime}}$;

2. $E_{\pi \cup\{q\}}$ and $E_{\pi^{\prime} \cup\{p\}}$, for all $p \in \pi, q \in \pi^{\prime}$.

(iii) G satisfies:

1. $E_{\pi}$ and $E_{\pi^{\prime}}$;

2. $E_{\{p, q\}}$, for all $p \in \pi, q \in \pi^{\prime}$.

We point out finally that the existence of Hall $\pi$-systems together with $\pi$-dominance do characterize $\pi$-separability, as we prove next.

Remark 2.9 We notice that for any group $G$ and any $\pi \subseteq \mathbb{P}$, the existence of complement $\pi$-bases is equivalent to the existence of Hall $\pi$-systems, and also to the existence of Sylow $\pi$-bases, by Proposition 2.4 and the corresponding constructions. In this case the group $G$ satisfies $E_{\pi}$ and $E_{\pi^{\prime}}$, and then, if $2 \in \pi, G$ satisfies $D_{\pi^{\prime}}$ (see [1]).

For any positive integer $n$, we denote by $\pi(n)$ the set of primes dividing $n$; for the order $|G|$ of a group $\mathrm{G}$, we set $\pi(G)=\pi(|G|)$.

Theorem 2.10 Assume that the group $G$ has a complement $\pi$-basis and satisfies $D_{\pi}$, where $2 \in \pi \subseteq \mathbb{P}$. Then $G$ is $\pi$-separable. 
Proof We argue by induction on the order of $G$. Let $N$ be a normal subgroup of $G$. By ([18, Theorem 7.7]), $G$ satisfies $D_{\pi}$ if and only if $N$ and $G / N$ satisfies $D_{\pi}$. We may then assume that $G$ is a simple group. If $\left|\pi(G) \cap \pi^{\prime}\right| \geq 2$, then $G$ would satisfy $E_{p^{\prime}}$ and $E_{q^{\prime}}$, with $p, q$ odd different primes dividing the order of $G$, which would imply that $G$ would not be simple by [1, Corollary 5.5]. Consequently we may assume that $\left|\pi(G) \cap \pi^{\prime}\right|=1$. On the other hand, by hypothesis and Remark $2.9, G$ satisfies $D_{\pi}$ and $D_{\pi^{\prime}}$, and we may assume that the group is neither a $\pi$-group nor a $\pi^{\prime}$-group. In [13, Lemma 3.1], such a simple group is characterized to be $G=P S L(2, q)$, where $q>3, q(q-1) \equiv 0(3), q \equiv-1(4)$ and $\pi(q+1) \subseteq \pi$, $\pi(q(q-1) / 2) \subseteq \pi^{\prime}$. Hence $\left|\pi(G) \cap \pi^{\prime}\right| \geq|\pi(q(q-1) / 2)| \geq 2$, which is not possible and proves that $G$ is $\pi$-separable.

\section{General framework: the theory of soluble groups}

Before focusing on the universe of $\pi$-separable groups, and proving our main results in Sect. 4, we present briefly here the general framework on the theory of soluble groups and classes of groups, where they are relevant to our concerns. It makes clear the origin of main concepts in our extension to $\pi$-separable groups, $\pi$ a set of primes, particularly the one of $\mathfrak{N}^{\pi}$-Dnormal subgroups, associated to the class $\mathfrak{N}^{\pi}$ of groups which are the direct product of a $\pi$-group and a nilpotent $\pi^{\prime}$-group.

We recall first some basic concepts and results, which are taken from [9]:

Definitions 3.1 [9, II. Sect. 2] A class $\mathfrak{X}$ of groups is a formation if every epimorphic image of a group in $\mathfrak{X}$ belongs to $\mathfrak{X}$, and $G /\left(N_{1} \cap N_{2}\right) \in \mathfrak{X}$ whenever $N_{1}, N_{2} \unlhd G$ with $G / N_{1}, G / N_{2} \in$ $\mathfrak{X}$.

In this case, the $\mathfrak{X}$-residual of a group $G$, denoted $G^{\mathfrak{X}}$, is the smallest normal subgroup of $G$ with quotient group in $\mathfrak{X}$ (which exists if $\mathfrak{X} \neq \emptyset$ ).

A formation $\mathfrak{X}$ is said to be saturated if $G \in \mathfrak{X}$ whenever $G / \Phi(G) \in \mathfrak{X}$, where $\Phi(G)$ denotes the Frattini subgroup of $G$.

For the class $\mathfrak{X}=\mathfrak{E}_{\pi}$ of all $\pi$-groups, $\pi$ a set of primes, we set $G^{\mathfrak{E}_{\pi}}=O^{\pi}(G)$ for the $\mathfrak{E}_{\pi}$-residual of the group $G$, also described as the subgroup generated by all $\pi^{\prime}$-subgroups of $G$.

Definitions 3.2 [9, III. Definitions (3.2), (3.5)(b)] Let $\mathfrak{X}$ be a class of groups, and $G$ be a group.

(a) A subgroup $U$ of $G$ is called an $\mathfrak{X}$-projector of $G$ if $U K / K$ is an $\mathfrak{X}$-maximal subgroup of $G / K$ (i.e. maximal as a subgroup of $G / K$ in $\mathfrak{X}$ ) for all $K \unlhd G$. The (possibly empty) set of $\mathfrak{X}$-projectors of $G$ will be denoted by $\operatorname{Proj}_{\mathfrak{X}}(G)$.

(b) An $\mathfrak{X}$-covering subgroup of $G$ is a subgroup $E$ of $G$ with the property that $E \in \operatorname{Proj}_{\mathfrak{X}}(H)$ whenever $E \leq H \leq G$. The set of $\mathfrak{X}$-covering subgroups of $G$ will be denoted by $\operatorname{Cov} \mathfrak{X}(G)$.

The classical theorem of Carter ( [7], [9, III. Theorem (4.6)]) states that each soluble group possesses exactly one conjugacy class of Carter subgroups, i.e. self-normalizing nilpotent subgroups, which coincide with $\mathfrak{N}$-projectors and with $\mathfrak{N}$-covering subgroups, for the class $\mathfrak{N}$ of nilpotent groups.

Definitions 3.3 [9, IV. Definitions (3.1), Theorem (3.2), II. Definition (1.2)(b)] 
(a) A formation function is a function $f$ which associates with each prime $p$ a (possibly empty) formation $f(p)$.

(b) A class $\mathfrak{F}$ of groups is called a local formation if there exists a formation function $f$ such that $\mathfrak{F}$ consists of the groups $G$ which satisfy that, for all chief factors $H / K$ of $G$ and for all primes $p$ dividing $|H / K|$, it holds that $G / C_{G}(H / K) \in f(p)$. The class $\mathfrak{F}$ is said to be locally defined by the formation function $f$ and denoted $\mathfrak{F}=L F(f)$.

The characteristic of the local formation $L F(f)$ is $\operatorname{Char}(L F(f))=\left\{p \in \mathbb{P} \mid Z_{p} \in\right.$ $L F(f)\}=\{p \in \mathbb{P} \mid f(p) \neq \emptyset\}$, where $Z_{p}$ denotes the cyclic group of order $p$.

Remark 3.4 [9, IV. Definition (3.9)(b)] A local formation $\mathfrak{F}$ always has a smallest local definition, i.e. a formation function $f$ such that $\mathfrak{F}=L F(f)$, and $f(p) \subseteq g(p)$ for every prime $p$ and any other formation function $g$ such that $\mathfrak{F}=\bar{L} F(\underline{f}) \overline{=} L F(g)$.

The well-known theorem of Gaschütz-Lubeseder-Schmid (cf. [9, IV. Theorem (4.6)] states that non-empty saturated formations are exactly local formations.

Let $\pi$ be a set of primes. Let

$$
\mathfrak{N}^{\pi}=\mathfrak{E}_{\pi} \times \mathfrak{N}_{\pi^{\prime}}=\left(G=H \times K \mid H \in \mathfrak{E}_{\pi}, K \in \mathfrak{N}_{\pi^{\prime}}\right),
$$

$\mathfrak{E}_{\pi}$ the class of all $\pi$-groups and $\mathfrak{N}_{\pi^{\prime}}$ the class of all nilpotent $\pi^{\prime}$-groups.

In the particular cases when either $\pi=\emptyset$ or $\pi=\{p\}, p$ a prime, $(|\pi| \leq 1)$, then $\mathfrak{N}^{\pi}=\mathfrak{N}$ is the class of all nilpotent groups.

Our main results Theorems 4.5 and 4.14 extend the existence and properties of Carter subgroups in soluble groups to $\rho$-separable groups, $\rho$ a set of primes, with appropriate class $\mathfrak{N}^{\rho}$ or $\mathfrak{N}^{\rho^{\prime}}$ playing the role of the class $\mathfrak{N}$ of nilpotent groups.

We shall appeal also to the concept of $\mathfrak{N}^{\pi}$-Dnormal subgroup, as $\mathfrak{N}^{\pi}$ is a saturated formation.

The concept of $\mathfrak{G}$-Dnormal subgroups for a non-empty saturated formation $\mathfrak{G}$, which was given by K. Doerk in the universe of finite soluble groups, and appears for the first time in [2, Definition 3.1], is also available for arbitrary finite groups, as defined next. For notation, if $G$ is a group, $\rho$ a set of primes, $G_{\rho} \in \operatorname{Hall}_{\rho}(G)$ and $H \leq G$, we write $G_{\rho} \searrow H$ to mean that $G_{\rho}$ reduces into $H$, i.e. $G_{\rho} \cap H \in \operatorname{Hall}_{\rho}(H)$.

Definition 3.5 [2, Definition 3.1] Let $\mathfrak{G}$ be a non-empty saturated formation and let $G$ be a group. A subgroup $H$ of $G$ is said to be $\mathfrak{G}$-Dnormal in $G$ if $\pi(|G: H|) \subseteq$ Char(G), and for every $p \in \operatorname{Char}(\mathfrak{G})$ it holds that

$$
\left[H_{G}^{p}, H^{\underline{g}(p)}\right] \leq H,
$$

where $\underline{g}$ denotes the smallest local definition of $\mathfrak{G}$ as local formation, and $H_{G}^{p}=\left\langle G_{p} \in\right.$ $\operatorname{Syl}_{p}\left(\overline{G)}\left|G_{p} \searrow H\right\rangle\right.$.

The saturated formation $\mathfrak{N}^{\pi}=\mathfrak{E}_{\pi} \times \mathfrak{N}_{\pi^{\prime}}=L F(f)=L F(f)$ is locally defined by the formation function $f$ given by $f(p)=\mathfrak{E}_{p}$ if $p \in \pi^{\prime}$, and $f(p)=\overline{\mathfrak{E}}_{\pi}$ if $p \in \pi$. Then the smallest local definition is given by $\underline{f}(p)=(1)$ if $p \in \pi^{\prime}$, and $\underline{f}(p)= \begin{cases}(1) & \text { if } \pi=\{p\}, \\ \mathfrak{E}_{\pi} & \text { if } p \in \pi,|\pi| \geq 2 .\end{cases}$

Hence, for $\mathfrak{N}^{\pi}=\mathfrak{N}, \mathfrak{N}$-Dnormal subgroups are exactly normal subgroups.

In the case $|\pi| \geq 2$, a subgroup $H$ of a group $G$ is $\mathfrak{N}^{\pi}$-Dnormal if it satisfies the following conditions:

(1) whenever $p \in \pi^{\prime}$ and $G_{p} \in \operatorname{Syl}_{p}(G), G_{p} \searrow H$, then $G_{p} \leq N_{G}(H)$; 
(2) whenever $p \in \pi$ and $G_{p} \in \operatorname{Syl}_{p}(G), G_{p} \searrow H$, then $G_{p} \leq N_{G}\left(O^{\pi}(H)\right)$.

Note that normal subgroups are $\mathfrak{N}^{\pi}$-Dnormal for any set of primes $\pi$.

Remark 3.6 Regarding the previous statement, note that for any $X \leq G$ it holds that $\left[X, O^{\pi}(H)\right] \leq H$ if and only if $X \leq N_{G}\left(O^{\pi}(H)\right)$.

Proof Assume that $\left[X, O^{\pi}(H)\right] \leq H$. We consider $O^{\pi}(H)=\left\langle H_{q}\right| H_{q} \in \operatorname{Syl}_{q}(H), q \in$ $\left.\pi^{\prime}\right\rangle$. Then $\left[X, O^{\pi}(H)\right] \leq\left\langle O^{\pi}(H)^{x} \mid x \in X\right\rangle=\left\langle H_{q}^{x} \mid x \in X, H_{q} \in \operatorname{Syl}_{q}(H), q \in \pi^{\prime}\right\rangle=$ $O^{\pi}(H)$. The converse is clear.

The next proposition provides a useful characterization of $\mathfrak{N}^{\pi}$-Dnormal subgroups.

Proposition 3.7 Let $H$ be a subgroup of a group $G$. Then:

1. Assume that $|\pi| \leq 1$. Then $\mathfrak{N}^{\pi}=\mathfrak{N}$ and $H$ is $\mathfrak{N}$-Dnormal in $G$ if and only $H$ is normal in $G$.

2. Assume that $|\pi| \geq 2$. Then the following statements are equivalent:

(i) $H$ is $\mathfrak{N}^{\pi}$-Dnormal in $G$;

(ii) $O^{\pi}(H) \unlhd G$ and $O^{\pi}(G) \leq N_{G}(H)$.

Proof Part 1 is clear. For Part 2, since $O^{\pi}(G)=\left\langle G_{p} \mid G_{p} \in \operatorname{Syl}_{p}(G), p \in \pi^{\prime}\right\rangle$, it is clear that (ii) implies (i). Conversely, assume the (i) holds, i.e. $H$ is $\mathfrak{N}^{\pi}$-Dnormal in $G$. By Sylow's theorem, for each prime $p$, there exists $G_{p} \in \operatorname{Syl}_{p}(G)$ such that $G_{p} \searrow H$. The definition of $\mathfrak{N}^{\pi}$-Dnormality implies that $G_{p} \leq N_{G}\left(O^{\pi}(H)\right)$ if $p \in \pi$, and $G_{p} \leq N_{G}(H) \leq$ $N_{G}\left(O^{\pi}(H)\right)$ if $p \in \pi^{\prime}$. Consequently, $G=\left\langle G_{p} \mid p \in \mathbb{P}\right\rangle \leq N_{G}\left(O^{\pi}(H)\right)$, i.e. $O^{\pi}(H) \unlhd G$. In particular, for any $p \in \pi^{\prime}$ and any $G_{p} \in \operatorname{Syl}_{p}(G)$, it holds that $G_{p} \cap H=G_{p} \cap O^{\pi}(H) \in$ $\operatorname{Syl}_{p}\left(O^{\pi}(H)\right)=\operatorname{Syl}_{p}(H)$, which means that $G_{p} \searrow H$, and then $G_{p} \leq N_{G}(H)$, because $H$ is $\mathfrak{N}^{\pi}$-Dnormal in $G$. Hence, $O^{\pi}(G)=\left\langle G_{p} \mid G_{p} \in \operatorname{Syl}_{p}(G), p \in \pi^{\prime}\right\rangle \leq N_{G}(H)$, and we are done.

For notation, whenever a group $X \in \mathfrak{N}^{\pi}$, we write $X=X_{\pi} \times X_{\pi^{\prime}}$ where $X_{\pi}=O_{\pi}(X) \in$ $\mathfrak{E}_{\pi}$ and $X_{\pi^{\prime}}=O_{\pi^{\prime}}(X) \in \mathfrak{N}_{\pi^{\prime}}$.

Corollary 3.8 Assume that $|\pi| \geq 2$ and let $H$ be a subgroup of a group $G$ such that $H=$ $H_{\pi} \times H_{\pi^{\prime}} \in \mathfrak{N}^{\pi}$. Then $H$ is $\mathfrak{N}^{\pi}$-Dnormal in $G$ if and only if $H_{\pi^{\prime}} \unlhd G$ and $O^{\pi}(G) \leq N_{G}(H)$.

Proof This is a consequence of Proposition 3.7(2) since in this case $H_{\pi^{\prime}}=O^{\pi}(H)$.

\section{Carter-like subgroups in $\pi$-separable groups}

Let $\pi$ be a set of primes. As above, set $\mathfrak{N}^{\pi}=\mathfrak{E}_{\pi} \times \mathfrak{N}_{\pi^{\prime}}$, where $\mathfrak{E}_{\pi}$ is the class of $\pi$-groups and $\mathfrak{N}_{\pi^{\prime}}$ is the class of nilpotent $\pi^{\prime}$-groups.

We prove in this section that if $G$ is a $\pi^{\prime}$-soluble group, then $\mathfrak{N}^{\pi}$-projectors coincide with $\mathfrak{N}^{\pi}$-covering subgroups, and they form a conjugacy class of self- $\mathfrak{N}^{\pi}$-Dnormalizing subgroups of $G$ (see Definition 4.7, and Theorems 4.5, 4.14). If $\mathfrak{N}^{\pi}=\mathfrak{N}$ is the class of nilpotent groups and $G$ is a soluble group, these are the Carter subgroups.

Remark 4.1 We notice that Burnside's $p^{a} q^{b}$-theorem together with the Feit-Thompson theorem imply that $\pi$-separable groups are $\pi^{\prime}$-soluble whenever $\left|\pi^{\prime}\right| \leq 2$, or $2 \in \pi$ if $\left|\pi^{\prime}\right| \geq 3$.

Also, by the Feit-Thompson theorem, for any set of primes $\rho$, a $\rho$-separable group is either $\rho$-soluble or $\rho^{\prime}$-soluble. 
For our main results we quote a series of results from [9, III. Sect. 3] and adhere to the notation there, though specialized to our saturated formation $\mathfrak{N}^{\pi}$ and our purposes. We notice that $b\left(\mathfrak{N}^{\pi}\right) \subseteq \mathcal{P}_{1} \cup \mathcal{P}_{2}$, where $b\left(\mathfrak{N}^{\pi}\right)$ is the class of groups $G \notin \mathfrak{N}^{\pi}$ but whose proper epimorphic images belong to $\mathfrak{N}^{\pi}$, and for each $i=1,2, \mathcal{P}_{i}$ is the class of primitive groups with a unique minimal normal subgroup, which is abelian for $i=1$, and non-abelian for $i=2$.

Lemma 4.2 1. [9, III. Proposition (3.7)] For a group $G$, whenever $N \unlhd G, N \leq V \leq G$, $U \in \operatorname{Proj}_{\mathfrak{N}^{\pi}}(V)$, and $V / N \in \operatorname{Proj}_{\mathfrak{N}^{\pi}}(G / N)$, then $U \in \operatorname{Proj}_{\mathfrak{N}^{\pi}}(G)$.

2. [9, III. Lemma (3.9)] Assume that $G \in b\left(\mathfrak{N}^{\pi}\right)$. Then:

(a) If $G \in \mathcal{P}_{1}$, then $\operatorname{Cov}_{\mathfrak{N}^{\pi}}(G)$ and $\operatorname{Proj}_{\mathfrak{N}^{\pi}}(G)$ both coincide with the non-empty set comprising those subgroups of $G$ which are complements in $G$ to the minimal normal subgroup of $G$.

(b) If $G \in \mathcal{P}_{2}$, then $\operatorname{Proj}_{\mathfrak{N}^{\pi}}(G)$ is non-empty and consists of all $\mathfrak{N}^{\pi}$-maximal subgroups of $G$ which supplement the minimal normal subgroup of $G$ in $G$.

3. [9, III. Theorem (3.10)] For any group $G, \operatorname{Cov}_{\mathfrak{N}^{\pi}}(G) \subseteq \operatorname{Proj}_{\mathfrak{N}^{\pi}}(G) \neq \emptyset$.

4. [9, III. Theorem (3.14)] Let $N$ be a nilpotent normal subgroup of a group $G$, and let $H$ be an $\mathfrak{N}^{\pi}$-maximal subgroup of $G$ such that $G=H N$. Then $H \in \operatorname{Proj}_{\mathfrak{N}^{\pi}}(G)$.

5. ([9, III. Theorem (3.19)], [11]) Let $\mathfrak{B}$ be the class of all $\pi^{\prime}$-soluble groups. The statement "Proj $\mathfrak{N}^{\pi}(G)$ is a conjugacy class of $G$ " is true for all groups $G \in \mathfrak{B}$ if and only if it is true for all $G \in b\left(\mathfrak{N}^{\pi}\right) \cap \mathfrak{B}$.

6. ([9, III. Remark (3.20)(b)], [11]) Let $\mathfrak{B}$ be the class of all $\pi^{\prime}$-soluble groups. The statement " $\operatorname{Proj}_{\mathfrak{N}^{\pi}}(G)=\operatorname{Cov}_{\mathfrak{N}^{\pi}}(G)$ " is true for all groups $G \in \mathfrak{B}$ if and only if it is true for all $G \in b\left(\mathfrak{N}^{\pi}\right) \cap \mathfrak{B}$.

We still quote the following result for our purposes.

Lemma 4.3 [5, Theorems 4.1.18, 4.2.17] Let $\mathfrak{H}$ be a saturated formation and let $G$ be a group whose $\mathfrak{H}$-residual $G^{\mathfrak{H}}$ is abelian. Then $G^{\mathfrak{H}}$ is complemented in $G$, any two complements are conjugate in $G$, and the complements are the $\mathfrak{H}$-projectors of $G$.

Lemma 4.4 Let $M=M_{\pi} \times M_{\pi^{\prime}}$ be an $\mathfrak{N}^{\pi}$-maximal subgroup of a $\pi$-separable group $G$. Then:

1. $M=M_{\pi^{\prime}} C_{G}\left(M_{\pi^{\prime}}\right)_{\pi}$ for some $C_{G}\left(M_{\pi^{\prime}}\right)_{\pi} \in \operatorname{Hall}_{\pi}\left(C_{G}\left(M_{\pi^{\prime}}\right)\right)$.

2. If $H=H_{\pi} \times H_{\pi^{\prime}}$ is another $\mathfrak{N}^{\pi}$-maximal subgroup of $G$ and $M_{\pi^{\prime}}^{x}=H_{\pi^{\prime}}$ for some $x \in G$, then $M^{g}=H$ for some $g \in G$.

Proof 1. We have that $M_{\pi} \leq C_{G}\left(M_{\pi^{\prime}}\right)$ and so $M_{\pi} \leq C_{G}\left(M_{\pi^{\prime}}\right)_{\pi}$ for some $C_{G}\left(M_{\pi^{\prime}}\right)_{\pi} \in$ Hall $_{\pi}\left(C_{G}\left(M_{\pi^{\prime}}\right)\right)$. But $M_{\pi^{\prime}} C_{G}\left(M_{\pi^{\prime}}\right)_{\pi} \in \mathfrak{N}^{\pi}$, which implies that $M=M_{\pi^{\prime}} C_{G}\left(M_{\pi^{\prime}}\right)_{\pi} \in$ $\mathfrak{N}^{\pi}$ by the maximality of $M$.

2. The hypothesis implies that $C_{G}\left(M_{\pi^{\prime}}\right)^{x}=C_{G}\left(M_{\pi^{\prime}}^{x}\right)=C_{G}\left(H_{\pi^{\prime}}\right)$. Then $M_{\pi}^{x}, H_{\pi} \in$ $\operatorname{Hall}_{\pi}\left(C_{G}\left(H_{\pi^{\prime}}\right)\right)$ and $M_{\pi}^{x y}=H_{\pi}$ for some $y \in C_{G}\left(H_{\pi^{\prime}}\right)$. Consequently, $M^{x y}=$ $M_{\pi}^{x y} M_{\pi^{\prime}}^{x y}=H_{\pi} H_{\pi^{\prime}}=H$, and we are done.

Theorem 4.5 If $G$ is a $\pi^{\prime}$-soluble group, then $\emptyset \neq \operatorname{Proj}_{\mathfrak{N}^{\pi}}(G)=\operatorname{Cov}_{\mathfrak{N}^{\pi}}(G)$ and it is a conjugacy class of $G$.

Proof By Lemma 4.2, parts (3), (5), (6), we may assume that $G \in b\left(\mathfrak{N}^{\pi}\right)$. Since $\mathfrak{N}^{\pi}$ is a saturated formation, $G \in \mathcal{P}_{1} \cup \mathcal{P}_{2}$; let $N$ be the minimal normal subgroup of $G$. 
If $G \in \mathcal{P}_{1}$, the result follows by Lemmas 4.2(2)(a) and 4.3.

Assume now that $G \in \mathcal{P}_{2}$. We know by Lemma 4.2(2)(b) that $\operatorname{Proj}_{\mathfrak{N}^{\pi}}(G)$ is non-empty and consists of all $\mathfrak{N}^{\pi}$-maximal subgroups of $G$ which supplement $N$ in $G$. We prove first that these subgroups are conjugate in $G$.

Let $M=M_{\pi} \times M_{\pi^{\prime}}, H=H_{\pi} \times H_{\pi^{\prime}}$ be $\mathfrak{N}^{\pi}$-maximal subgroups of $G$ such that $G=$ $N M=N H$. Since $G$ is $\pi^{\prime}$-soluble, and $N$ is non-abelian, $N$ is a $\pi$-group and, consequently, $M_{\pi^{\prime}}, H_{\pi^{\prime}} \in \operatorname{Hall}_{\pi^{\prime}}(G)$. Hence, there exists $x \in G$ such that $M_{\pi^{\prime}}^{x}=H_{\pi^{\prime}}$ and $M$ and $H$ are conjugate by Lemma 4.4(2).

We claim now that $\operatorname{Proj}_{\mathfrak{N}^{\pi}}(G) \subseteq \operatorname{Cov}_{\mathfrak{N}^{\pi}}(G)$, which will conclude the proof.

Let $M \in \operatorname{Proj}_{\mathfrak{N}^{\pi}}(G)$, i.e. $M=M_{\pi} \times M_{\pi^{\prime}}$ is an $\mathfrak{N}^{\pi}$-maximal subgroup of $G$ such that $G=N M$. Let $M \leq L \leq G$. We aim to prove that $M \in \operatorname{Proj}_{\mathfrak{N}^{\pi}}(L)$. Let $T \in \operatorname{Proj}_{\mathfrak{N}^{\pi}}(L)$. We notice that $L=M(L \cap N)$. Then $L /(L \cap N) \cong M /(M \cap N) \in \mathfrak{N}^{\pi}$. Since $T(L \cap N) /(L \cap N)$ is $\mathfrak{N}^{\pi}$-maximal in $L /(L \cap N)$, it follows that $L=T(L \cap N)$. Hence $T_{\pi^{\prime}}, M_{\pi^{\prime}} \in \operatorname{Hall}_{\pi^{\prime}}(L)$ and, moreover, $T$ and $M$ are $\mathfrak{N}^{\pi}$-maximal subgroups of $L$. By Lemma 4.4(2), $T$ and $M$ are conjugate in $L$ and $M \in \operatorname{Proj}_{\mathfrak{N}^{\pi}}(L)$.

Remark 4.6 In Theorem 4.5 the hypothesis of $\pi^{\prime}$-solubility cannot be weakened to $\pi$ separability. Otherwise, for the particular case when $\pi=\emptyset$, the result would hold for every finite group and the formation $\mathfrak{N}^{\pi}=\mathfrak{N}$ of nilpotent groups, which is not true. Particularly, also if $\pi \neq \emptyset$, one can consider for instance $\pi=\mathbb{P}-\{2,3,5\}, \pi^{\prime}=\{2,3,5\}$ and $G=\operatorname{Alt}(5)$ the alternating group of degree 5 . The group $G$ is obviously $\pi$-separable, the $\mathfrak{N}^{\pi}$-projectors are the $\mathfrak{N}$-projectors, which do not form a conjugacy class of subgroups, as they are all the Sylow subgroups of $G$; and $G$ has no $\mathfrak{N}$-covering subgroups.

Definition 4.7 A subgroup $H$ of a group $G$ is said to be self- $\mathfrak{N}^{\pi}$-Dnormalizing in $G$ if whenever $H \leq K \leq G$ and $H$ is $\mathfrak{N}^{\pi}$-Dnormal in $K$, then $H=K$.

We prove next that $\mathfrak{N}^{\pi}$-projectors are self- $\mathfrak{N}^{\pi}$-Dnormalizing subgroups.

Proposition 4.8 Let $H$ be an $\mathfrak{N}^{\pi}$-projector of a $\pi^{\prime}$-soluble group $G$. Then $H$ is self- $\mathfrak{N}^{\pi}$ Dnormalizing in $G$.

Proof Assume that $H \leq K \leq G$ and $H$ is $\mathfrak{N}^{\pi}$-Dnormal in $K$. We aim to prove that $H=K$. By Theorem 4.5 and Corollary 3.8, we have that $H \in \operatorname{Cov}_{\mathfrak{N}^{\pi}}(K)$ and $H_{\pi^{\prime}} \unlhd K$. Then $H / H_{\pi^{\prime}} \in \operatorname{Proj}_{\mathfrak{N}^{\pi}}\left(K / H_{\pi^{\prime}}\right)$ and $H / H_{\pi^{\prime}} \leq K_{\pi} H_{\pi^{\prime}} / H_{\pi^{\prime}} \in \mathfrak{N}^{\pi}$, for any $K_{\pi} \in \operatorname{Hall}_{\pi}(K)$, which implies that $H=K_{\pi} H_{\pi^{\prime}}$. Whence, if $K_{\pi^{\prime}} \in \operatorname{Hall}_{\pi^{\prime}}(K)$, then $K=H K_{\pi^{\prime}}$ and $H \unlhd K$ by Proposition 3.7. If $H<K$, then $H<H K_{p}$ for some $p \in \pi^{\prime}$ and $1 \neq K_{p} \in \operatorname{Syl}_{p}(K)$. But $H K_{p} / H \in \mathfrak{N}^{\pi}$ which contradicts the fact that $H \in \operatorname{Cov}_{\mathfrak{N}^{\pi}}(K)$.

Lemma 4.9 Assume that $|\pi| \geq 2, H=H_{\pi} \times H_{\pi^{\prime}}, K=K_{\pi} \times K_{\pi^{\prime}} \in \mathfrak{N}^{\pi}$ and $H \leq K$. Then $H$ is $\mathfrak{N}^{\pi}$-Dnormal in $K$ if and only if $H_{\pi^{\prime}} \unlhd K_{\pi^{\prime}}$.

Proof We notice that $\left[K_{\pi}, H_{\pi^{\prime}}\right]=1$ and $\left[K_{\pi^{\prime}}, H_{\pi}\right]=1$. Consequently, Corollary 3.8 implies that $H$ is $\mathfrak{N}^{\pi}$-Dnormal in $K$ if and only if $H_{\pi^{\prime}} \unlhd K_{\pi^{\prime}}$.

Proposition 4.10 Assume that $H=H_{\pi} \times H_{\pi^{\prime}}<L=L_{\pi} \times L_{\pi^{\prime}} \in \mathfrak{N}^{\pi}$. Then there exists $K \leq L$ such that $H<K$ and $H$ is $\mathfrak{N}^{\pi}$-Dnormal in $K$.

Proof If $|\pi| \leq 1$, then $\mathfrak{N}^{\pi}=\mathfrak{N}$, and the result is clear. In the case $|\pi| \geq 2$, by Lemma 4.9, if $H_{\pi^{\prime}}=L_{\pi^{\prime}}$, then $H$ is $\mathfrak{N}^{\pi}$-Dnormal in $L$, and we are done. Otherwise, there exists $T \leq L_{\pi^{\prime}}$ such that $H_{\pi^{\prime}} \triangleleft T$, i.e. $H_{\pi^{\prime}}$ is a proper normal subgroup of $T$, because $L_{\pi^{\prime}}$ is nilpotent. We can consider now the subgroup $K=L_{\pi} T \leq L$ which satisfies that $H<K$ and $H$ is $\mathfrak{N}^{\pi}$-Dnormal in $K$, which concludes the proof. 
As a consequence we can state the following:

Corollary 4.11 If $H \in \mathfrak{N}^{\pi}$ is a self- $\mathfrak{N}^{\pi}$-Dnormalizing subgroup of a group $G$, then $H$ is $\mathfrak{N}^{\pi}$-maximal in $G$.

Remark 4.12 It is not true in general that $\mathfrak{N}^{\pi}$-projectors of $\pi^{\prime}$-soluble groups are exactly self- $\mathfrak{N}^{\pi}$-Dnormalizing subgroups in $\mathfrak{N}^{\pi}$. Otherwise, $\mathfrak{N}^{\pi} \cap \mathfrak{S}$ would be either $\mathfrak{N}$ or $\mathfrak{S}$, the class of all soluble groups, by [3, Proposition 4.1]. But we see next that a corresponding result to [3, Theorem 4.2] is still possible. That reference provides a corresponding result to our next Theorem 4.14, for finite soluble groups, subgroup-closed saturated formations and associated projectors.

As a consequence of Lemma 4.3 we can state the following.

Lemma 4.13 Let $\mathfrak{H}$ be a saturated formation, $X$ be a group and $H$ be an $\mathfrak{H}$-projector of $X$. Then $H \cap X^{\mathfrak{H}} \leq\left(X^{\mathfrak{H}}\right)^{\prime}$.

Theorem 4.14 For a subgroup $H$ of a $\pi^{\prime}$-soluble group $G$ the following statements are pairwise equivalent:

1. $H$ is an $\mathfrak{N}^{\pi}$-projector of $G$.

2. $H$ is an $\mathfrak{N}^{\pi}$-covering subgroup of $G$.

3. $H \in \mathfrak{N}^{\pi}$ is a self- $\mathfrak{N}^{\pi}$-Dnormalizing subgroup of $G$ and $H$ satisfies the following property:

$$
\text { If } H \leq X \leq G, \text { then } H \cap X^{\mathfrak{N}^{\pi}} \leq\left(X^{\mathfrak{N}^{\pi}}\right)^{\prime} .
$$

Proof The equivalence $1 \leftrightarrow 2$ has been proven in Theorem 4.5. On the other hand, Proposition 4.8 and Lemma 4.13 prove $2 \rightarrow 3$. We prove next that $3 \rightarrow 1$.

Let $H \in \mathfrak{N}^{\pi}$ be a self- $\mathfrak{N}^{\pi}$-Dnormalizing subgroup of $G$ satisfying property (*). We aim to prove that $H \in \operatorname{Proj}_{\mathfrak{N}^{\pi}}(G)$. We notice that $H$ is $\mathfrak{N}^{\pi}$-maximal in $G$ by Corollary 4.11.

If $G \in \mathfrak{N}^{\pi}$, then $H=G$ and the result follows. So that we may assume that $G \notin \mathfrak{N}^{\pi}$. We argue by induction on the order of $G$. Let $N$ be a minimal normal subgroup of $G$ such that $N \leq G^{\mathfrak{N}^{\pi}}$.

We distinguish the following cases:

Case 1. $G=H N$.

Case 2. $H N<G$.

Case 1. If $N$ is abelian, the result follows by Lemma 4.2(4). Assume that $N$ is not abelian. Let $K \unlhd G$ such that $G / K \in b\left(\mathfrak{N}^{\pi}\right)$. Then $N$ is not contained in $K$ because $G / N \cong$ $H /(H \cap N) \in \mathfrak{N}^{\pi}$. In particular, $N \cap K=1$ and $N \cong N K / K$ is a minimal normal subgroup of $G / K=(N K / K)(H K / K) \in \mathcal{P}_{2}$, with $H K / K<G / K$ because $H K / K \in$ $\mathfrak{N}^{\pi}$. By Lemma 4.2(2)(b), $H K / K \leq P / K$ for some $P / K \in \operatorname{Proj}_{\mathfrak{N}^{\pi}}(G / K)$. We have now that $H \leq P<G$. The inductive hypothesis implies that $H \in \operatorname{Proj}_{\mathfrak{N}^{\pi}}(P)$, and from Lemma 4.2(1), $H \in \operatorname{Proj}_{\mathfrak{N}^{\pi}}(G)$, as claimed.

Case 2. In this case $H N<G$ and the inductive hypothesis implies that $H \in \operatorname{Proj}_{\mathfrak{N}^{\pi}}(H N)$. We prove first that $H N / N$ satisfies property $\left({ }^{*}\right)$ in $G / N$. Assume that $H N / N \leq$ $X / N \leq G / N$. If $X<G$, then $H \in \operatorname{Proj}_{\mathfrak{N}^{\pi}}(X)$ by inductive hypothesis and then $H N / N \cap(X / N)^{\mathfrak{N}^{\pi}} \leq\left((X / N)^{\mathfrak{N}^{\pi}}\right)^{\prime}$. Otherwise, $X=G$ and so $(H N / N) \cap(X / N)^{\mathfrak{N}^{\pi}}=$ $(H N / N) \cap(G / N)^{\mathfrak{N}^{\pi}}=(H N / N) \cap G^{\mathfrak{N}^{\pi}} / N=\left(H \cap G^{\mathfrak{N}^{\pi}}\right) N / N \leq\left(G^{\mathfrak{N}^{\pi}}\right)^{\prime} N / N=$ $\left((G / N)^{\mathfrak{N}^{\pi}}\right)^{\prime}=\left((X / N)^{\mathfrak{N}^{\pi}}\right)^{\prime}$. 
We claim that $H N / N \in \mathfrak{N}^{\pi}$ is self- $\mathfrak{N}^{\pi}$-Dnormalizing in $G / N$. Then the result follows by inductive hypothesis together with Lemma 4.2(1).

Assume that $H N / N$ is $\mathfrak{N}^{\pi}$-Dnormal in $L / N \leq G / N$.

If $L<G$, the inductive hypothesis implies that $H$ is an $\mathfrak{N}^{\pi}$-projector of $L$ and so $H N=L$ by Proposition 4.8. So that we may assume that $L=G$ and $H N / N$ is $\mathfrak{N}^{\pi}$-Dnormal in $G / N$.

We split the rest of the proof into the following steps:

Step 1. $N_{G}(H N)=H N$.

If $g \in N_{G}(H N)$, then $H N=H^{g} N$. Since $H \in \operatorname{Proj}_{\mathfrak{N}^{\pi}}(H N)$, and this is a conjugacy class of subgroups of $H N$ by Theorem 4.5, $H^{g}=H^{x}$ for some $x \in H N$. Consequently, $g x^{-1} \in N_{G}(H)=H$, and $g \in H N$.

Step 2. $O^{\pi}(G) \leq H N$.

Since $H N / N$ is $\mathfrak{N}^{\pi}$-Dnormal in $G / N$, we have by Proposition 3.7 and Step 1 that $O^{\pi}(G) N / N \leq N_{G / N}(H N / N)=H N / N$.

Step 3. $O^{\pi}(G) H=H N$ and it is a maximal subgroup of $G$.

Assume that $O^{\pi}(G) \leq H N \leq T<G$. The inductive hypothesis implies that $H \in$ $\operatorname{Proj}_{\mathfrak{N}^{\pi}}(T)$. Moreover, $T / O^{\pi}(G) \in \mathfrak{E}_{\pi} \subseteq \mathfrak{N}^{\pi}$. Hence $T=O^{\pi}(G) H=H N$.

Step 4. $G / N=\left(H_{\pi^{\prime}} N / N\right) \times O_{\pi}(G / N) \in \mathfrak{N}^{\pi}$. In particular, $G^{\mathfrak{N}^{\pi}}=N$.

We have that $H_{\pi^{\prime}} N / N \unlhd G / N$ and $H_{\pi^{\prime}} N / N \in \operatorname{Hall}_{\pi^{\prime}}(G / N)$ by Corollary 3.8 and Step 2 . Since $\left[H_{\pi}, H_{\pi^{\prime}}\right]=1$ and $G / N$ is $\pi$-separable, and equivalently $\pi^{\prime}$-separable, it follows by $[14,6$. Theorem 3.2] that

$$
\begin{aligned}
\left(H_{\pi} N / N\right) O_{\pi}(G / N) / O_{\pi}(G / N) & \leq C_{(G / N) / O_{\pi}(G / N)}\left(O_{\pi^{\prime}}\left((G / N) / O_{\pi}(G / N)\right)\right) \\
& \leq O_{\pi^{\prime}}\left((G / N) / O_{\pi}(G / N)\right),
\end{aligned}
$$

which implies $H_{\pi} N / N \leq O_{\pi}(G / N)$.

If $H_{\pi} N / N=O_{\pi}(G / N)$, then $H N / N \unlhd G / N$ and $G=H N$ by Step 1 , a contradiction.

Consequently, we may assume that $H_{\pi} N / N<O_{\pi}(G / N)$. Then $H N / N<$ $\left(H_{\pi^{\prime}} N / N\right) O_{\pi}(G / N) \leq G / N$. By Step 3, $\left(H_{\pi^{\prime}} N / N\right) O_{\pi}(G / N)=G / N \in \mathfrak{N}^{\pi}$.

Step 5. $G=N P$ where $P=P_{\pi} \times P_{\pi^{\prime}} \in \operatorname{Proj}_{\mathfrak{N}^{\pi}}(G)$.

It follows by Step 4.

Step 6. $N \in \mathfrak{E}_{\pi^{\prime}}$.

If $N \in \mathfrak{E}_{\pi}$, then $H_{\pi^{\prime}}, P_{\pi^{\prime}} \in \operatorname{Hall}_{\pi^{\prime}}(G)$. Since $H$ and $P$ are both $\mathfrak{N}^{\pi}$-maximal subgroups, it follows by Lemma 4.4 that $H=P^{x} \in \operatorname{Proj}_{\mathfrak{N}^{\pi}}(G)$ and then $H N=G$, a contradiction. Since $G$ is $\pi$-separable, $N \in \mathfrak{E}_{\pi^{\prime}}$.

Step 7. Final contradiction.

Steps 4, 6, imply that $N=G^{\mathfrak{N}^{\pi}}$ is abelian, since $G$ is $\pi^{\prime}$-soluble. By hypothesis, $H \cap N=$ 1. Moreover, $P_{\pi^{\prime}} N=H_{\pi^{\prime}} N=O_{\pi^{\prime}}(G)$ and $P_{\pi} \in \operatorname{Hall}_{\pi}(G)$. There is no loss of generality to assume that $H_{\pi} \leq P_{\pi}$ and then $\left[H_{\pi}, P_{\pi^{\prime}}\right]=1$.

Since $H$ is an $\mathfrak{N}^{\pi}$-maximal subgroup of $H N=H_{\pi} H_{\pi^{\prime}} N$, we deduce that $H_{\pi^{\prime}}$ is an $\mathfrak{N}$ maximal subgroup of $C_{H_{\pi^{\prime}} N}\left(H_{\pi}\right)=H_{\pi^{\prime}} C_{N}\left(H_{\pi}\right)$. But $C_{N}\left(H_{\pi}\right) \unlhd C_{H_{\pi^{\prime}} N}\left(H_{\pi}\right)$ and $C_{N}\left(H_{\pi}\right)$ is nilpotent. By Lemma 4.2(4), $H_{\pi^{\prime}} \in \operatorname{Proj}_{\mathfrak{N}}\left(C_{H_{\pi^{\prime}} N}\left(H_{\pi}\right)\right)$. On the other hand,

$$
C_{H_{\pi^{\prime} N}}\left(H_{\pi}\right)=C_{P_{\pi^{\prime}} N}\left(H_{\pi}\right)=P_{\pi^{\prime}} C_{N}\left(H_{\pi}\right)
$$

Since $P_{\pi^{\prime}}$ is nilpotent, it follows again by Lemma 4.2(4), that $P_{\pi^{\prime}} \leq H_{\pi^{\prime}}^{x} \in$ $\operatorname{Proj}_{\mathfrak{N}}\left(C_{H_{\pi^{\prime}} N}\left(H_{\pi}\right)\right)$, for some $x \in C_{N}\left(H_{\pi}\right)$. If $P_{\pi^{\prime}}<H_{\pi^{\prime}}^{x}$, since $P_{\pi^{\prime}} N=H_{\pi^{\prime}}^{x}, N$, we have that $H_{\pi^{\prime}}^{x} \cap N \neq 1$ and so also $H \cap N \neq 1$, a contradiction. Therefore, $P_{\pi^{\prime}}=H_{\pi^{\prime}}^{x}$, and Lemma 4.4 implies that $H^{g}=P$ for some $g \in G$ as $H$ and $P$ are $\mathfrak{N}^{\pi}$-maximal subgroups of $G$. It follows that $H \in \operatorname{Proj}_{\mathfrak{N}^{\pi}}(G)$ and $H N=G$, the final contradiction. 
Acknowledgements The authors want to thank Peter Hauck for helpful conversations. This research has been supported by Proyectos PROMETEO/2017/ 057 from the Generalitat Valenciana (Valencian Community, Spain), and PGC2018-096872-B-I00 from the Ministerio de Ciencia, Innovación y Universidades, Spain, and FEDER, European Union. The fourth author acknowledges with thanks the financial support of the Universitat de València as research visitor (Programa Propi d'Ajudes a la Investigació de la Universitat de València, Subprograma Atracció de Talent de VLC-Campus, Estades d'investigadors convidats (2019)).

Funding Open Access funding provided thanks to the CRUE-CSIC agreement with Springer Nature.

Open Access This article is licensed under a Creative Commons Attribution 4.0 International License, which permits use, sharing, adaptation, distribution and reproduction in any medium or format, as long as you give appropriate credit to the original author(s) and the source, provide a link to the Creative Commons licence, and indicate if changes were made. The images or other third party material in this article are included in the article's Creative Commons licence, unless indicated otherwise in a credit line to the material. If material is not included in the article's Creative Commons licence and your intended use is not permitted by statutory regulation or exceeds the permitted use, you will need to obtain permission directly from the copyright holder. To view a copy of this licence, visit http://creativecommons.org/licenses/by/4.0/.

\section{References}

1. Arad, Z., Fisman, E.: On finite factorizable groups. J. Algebra 86, 522-548 (1984)

2. Arroyo-Jordá, M., Pérez-Ramos, M.D.: On the lattice of $\mathfrak{F}$-Dnormal subgroups in finite soluble groups. J. Algebra 242, 198-212 (2001)

3. Arroyo-Jordá, M., Pérez-Ramos, M.D.: Fitting classes and lattice formations I. J. Aust. Math. Soc. 76, 93-108 (2004)

4. Arroyo-Jordá, M., Arroyo-Jordá, P., Dark, R., Feldman, A.D., Pérez-Ramos, M.D.: Injectors in $\pi$ separable groups. Mediterranean J. Math. (to appear)

5. Ballester-Bolinches, A., Ezquerro, L.M.: Classes of finite groups. In: Mathematics and Its Applications, vol. 584. Springer, New York (2006)

6. Čunihin, S.A.: On theorems of Sylow's type. Doklady Akad. Nauk. SSSR 66, 165-168 (1949)

7. Carter, R.W.: Nilpotent self-normalizing subgroups of soluble groups. Math. Z. 75, 136-139 (1961)

8. Carter, R.W., Hawkes, T.O.: The F-normalizers of a finite soluble group. J. Algebra 5, 175-201 (1967)

9. Doerk, K., Hawkes, T.: Finite soluble groups. De Gruyter (1992)

10. Du, Z.: Hall subgroups and $\pi$-separable groups. J. Algebra 195, 501-509 (1997)

11. Förster, P.: Projektive Klassen endlicher Gruppen I. Schunck- und Gaschützklassen. Math. Z. 186, 149178 (1984)

12. Gaschütz, W.: Zur Theorie der endlichen auflösbaren Gruppen. Math. Z. 80, 300-305 (1963)

13. Gilotti, A.L.: $D_{\pi}$-property and normal subgroups. Ann. Mat. Pura Appl. 148, 227-235 (1987)

14. Gorenstein, D.: Finite groups. Chelsea Publishing Company (1980)

15. Hall, P.: A note on soluble groups. J. Lond. Math. Soc. 3, 98-105 (1928)

16. Hall, P.: A characteristic property of soluble groups. J. Lond. Math. Soc. 12, 188-200 (1937)

17. Hawkes, T.O.: On formation subgroups of a finite soluble group. J. Lond. Math. Soc. 44, 243-250 (1969)

18. Revin, D.O., Vdovin, E.P.: Hall subgroups in finite groups. In: Ischia Group Theory 2004, Contemp. Math. 402, (Amer. Math. Soc., Providence, RI, 2006), pp. 229-263

19. Vdovin, E.P.: Carter subgroups of finite almost simple groups. Algebra Logika 46(2), 157-216 (2007)

Publisher's Note Springer Nature remains neutral with regard to jurisdictional claims in published maps and institutional affiliations. 